\title{
VACANCIES IN PURE ICE STUDIED BY POSITRON ANNIHILATION TECHNIQUES
}

\author{
By O. E. Mogensen and M. Eldrup \\ (Chemistry Department, Risø National Laboratory, 40oo Roskilde, Denmark)
}

\begin{abstract}
Positron annihilation techniques (PAT) are briefly discussed, and the information that may be obtained about the positronium (Ps) states is compared to that obtainable about the similar electron states. The behaviour of Ps in monocrystals of pure light and heavy ice was studied at temperatures between $-185^{\circ} \mathrm{C}$ and the melting point. Ps is very probably trapped in vacancies (i.e. missing water molecules) in ice. A vacancy formation energy of roughly $0.2-0.35 \mathrm{eV}$ was derived in agreement with the value $0.28 \pm 0.07$ $\mathrm{eV}$ obtained previously from studies of the annealing of irradiated ice. The vacancy concentration is at least a few parts per million at the melting point, i.e. roughly $10^{4}$ times higher than normally assumed in the literature. The fact that the vacancy concentration is comparable to that of the hydrogen-bond defects will probably enforce a pronounced change in the "hydrogen-bond-defects" theory of the electric properties of ice.

RÉsumé. Étude des lacunes dans la glace pure par les techniques d'annihilation de positon. Les techniques d'annihilation de positon sont brèvement discutées et l'information qu'on peut obtenir sur les états de positonium (Ps) est comparée avec cela pour les états électroniques semblables. Le comportement de Ps dans les monocristaux de glace pure normale et lourde est étudié entre $-185^{\circ} \mathrm{C}$ et le point de fusion. Le Ps est très probablement piégé dans les lacunes (c'est-à-dire les molécules d'eau absentes de leur site) dans la glace. Nous obtenons une énergie de formation des lacunes comprise entre $0,2 \mathrm{et} 0.35 \mathrm{eV}$ en accord avec la valeur de $0,28 \pm 0,07 \mathrm{eV}$ obtenue antérieurement par les études de recuit de la glace irradiée. La concentration en lacunes est au moins de quelques p.p.m. au point de fusion c'est-à-dire sensiblement $10^{4}$ fois plus grande que la valeur annoncée dans la littérature. Le fait que cette concentration en lacunes est comparable à celle des défauts sur les liaisons hydrogène renforcera probablement le nécessité de modifier l'interprétation théorique des propriétés électriques reposant généralement sur la simple notion de défauts rotationnels.

Zusammenfassung. Leerstellen in reinem Eis, untersucht mit Hilfe von Positronzerstrahlungstechniken. Positronzerstrahlungstechniken (PAT) werden erörtert, und die Kenntnis, die man über die Positronium-(Ps) zustände gewinnt, wird mit der über die ähnlichen Elektronenzustände verglichen. Das Verhalten von Ps in Einkristallen von reinem, leichten und schweren Eis wurde bei Temperaturen zwischen $-185^{\circ} \mathrm{C}$ und dem Schmelzpunkt untersucht. Ps ist sehr wahrscheinlich in Leerstellen (d.h. fehlenden Wassermolekülen) im Eis eingefangen. Es wurde eine Leerstellenbildungsenergie von rund 0,2 bis $0,35 \mathrm{eV}$ abgeleitet, in guter Übereinstimmung mit dem Wert $0,34 \pm 0,07 \mathrm{eV}$, der früher aus der Untersuchung des Ausheilens von strahlungsgeschädigtem Eis gewonnen worden war. Die Leerstellenkonzentration beträgt am Schmelzpunkt mindestens einige je Million, d.h. ungefähr $1^{4}$-mal höher als üblicherweise in der Literatur angenommen. Die Tatsache, dass die Leerstellenkonzentration mit derjenigen der Wasserstoffbindungsfehler vergleichbar ist, wird wahrscheinlich eine deutliche Änderung in der Theorie der elektrischen Eigenschaften von Eis bewirken, die auf Fehlstellen in den Wasserstoff brücken beruht.
\end{abstract}

\section{INTRODUCTION}

The electron, the positron, and the positronium $(\mathrm{Ps})$ atom are the only light particles that participate in the low-energy processes of physics and chemistry. Because of their small masses, quantum-mechanical phenomena such as zero-point motion, tunnelling, delocalization, etc., are of great importance for the description of their behaviour. The positron is the antiparticle of the electron, hence it has the same mass as the electron, but it is oppositely charged. The Ps atom is the hydrogen-like, electron-positron bound state.

An important method of studying defects in solids is to measure the properties of electrons bound to the defects. Well-known examples are the recording of the absorption spectra of F-centres in alkali halides, and of electrons trapped in defects in amorphous and crystalline ice. Information on defects in metals and in ice can also be obtained through studies of their influence on the conductivity resulting from mobile electrons. Similarly, information on defects in solids can be obtained by measuring the properties of positrons and Ps atoms that get trapped in the defects (West, r 973).

A positron entering a solid or a liquid will normally be slowed down to thermal velocities within a very short time (typically $<\mathrm{I}$ ps). After a period (the positron lifetime) it will annihilate with an electron and two $\gamma$-quanta are normally emitted. These annihilation 
photons carry information about the electron-positron state at the moment of annihilation. Hence, experimental studies of the photons may give useful information about the various positron processes that precede the annihilation. Some of the positrons form Ps before the annihilation in various molecular liquids and solids and in some ionic crystals, but not in metals and semiconductors. In ice, roughly $74 \%$ of the positrons form Ps.

At defect concentrations above roughly parts per million (p.p.m.), the positron or Ps atom may get trapped in the defects before the annihilation, whereby the properties of the annihilation photons change. The trapping of positrons in "hole"-type defects (vacancies, voids, etc.) in metals has recently developed into an important new tool in metal-defect studies. Apparently, information on small vacancy clusters (typically $<50-500$ vacancies) that cannot be resolved by electron microscopy, can only be obtained by the positron-trapping method. Trapping of Ps in "hole"-type defects in solids has been used very little in defect studies. Apparently, ice is the only substance that has been investigated in detail as yet. In ice at $-\mathrm{I}^{\circ} \mathrm{C}$ roughly $65 \%$ of the Ps atoms are trapped in defects (mainly vacancies, i.e. missing water molecules) before the annihilation.

The purpose of the present paper is briefly to discuss the positron annihilation technique (PAT) in general and the results of our Ps trapping studies of vacancies, etc., in pure ice. The studies of vacancies in HF-doped, and irradiated ice are discussed in another paper at this conference (Eldrup and others, 1978). The work described here has been or will be published in full detail in a series of papers on positron annihilation in ice and related structures (Petersen and others, I970; Mogensen and others, I971 ; Milošević-Kvajić and others, I972 ; Eldrup and others, I972; Mogensen and Eldrup, I973; Eldrup, r976; Mogensen and Eldrup, I977[a], [b]; Eldrup and others, 1978; and a paper in preparation by M. Eldrup, R. J. Douglas, and L. Lupton).

Our ice results are of general interest from several points of view. First, the particular features of the annihilation process allow a direct determination of the Fourier transform of the Ps centre-of-mass wave function. Such detailed information is not available for other particles (e.g. the electrons in Bloch-function or trapped states). Secondly, Ps is in a Bloch-function state in the defect-free ice; apparently, Ps is the only atom shown to be in a Bloch-function state. Thirdly, the change in the wave function due to the introduction of vacancies in ice, i.e. the change from a mainly delocalized to a mainly localized Ps state, has been studied. Fourthly, information on vacancies and polyvacancies in ice has been obtained; very little is known about vacancies and polyvacancies in any molecular crystal. However, we shall in this paper emphasize the defect aspects of our work.

In the next section a general discussion of PAT and the trapping of Ps is given. The following section contains a short description of our results for pure ice and a discussion of the information obtained on vacancies in ice.

\section{General discussion of PAT}

The lifetimes of the positron and the Ps atom in liquids and solids are so short (o. I $-5 \mathrm{~ns}$ ) that at most only a few species are present in a sample. Hence, the methods used to investigate electrons in solids (e.g. light absorption, EPR, conductivity) cannot be applied in the positron case. The only way to get information on the behaviour of the positron or the Ps atom is to investigate the annihilation photons by use of the methods of gamma-ray spectroscopy. The principles of the two most important experimental techniques are illustrated in Figures I and 2 (Goldanskii, I968; West, I973). In a positron lifetime measurement ${ }^{22} \mathrm{Na}$ is normally used as positron source $(\approx 30 \mu \mathrm{Ci})$. The detection of a $1.28 \mathrm{MeV}$ photon emitted by this isotope almost simultaneously with the positron tells us that a positron has entered the sample, while the annihilation of the positron is signalled by the detection of one of the annihilation photons (usually with 0.5 I I MeV of energy). The time interval between the two signals is the positron 


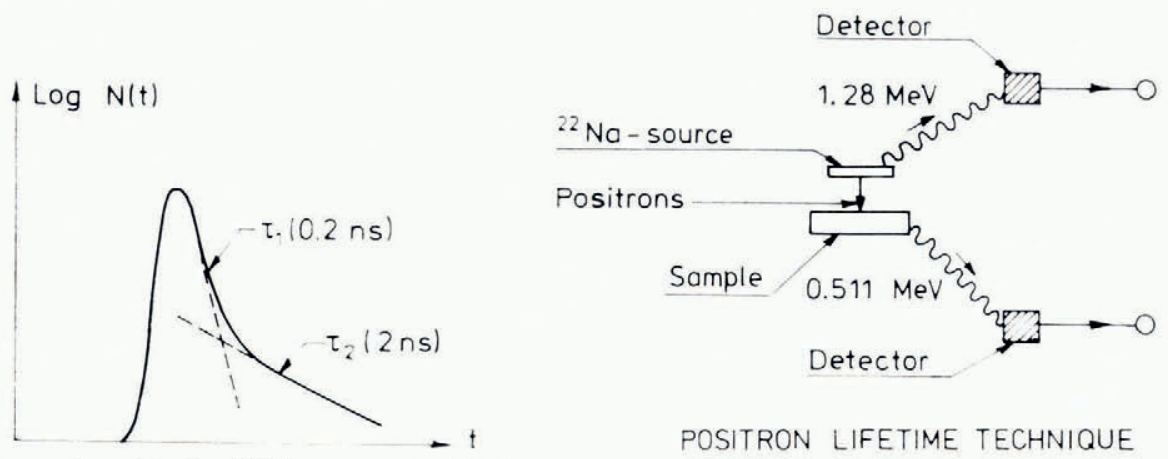

Fig. I. A two-component positron lifetime spectrum and a diagram of the experimental set-up for positron lifetime measurements.

lifetime. A typical lifetime distribution, as shown in Figure I, normally consists of a sum of several exponentially decaying components with mean lifetimes $\tau_{i}$ (normally just called lifetimes) and relative intensities $I_{i}$. Typical measuring times are $2-20 \mathrm{~h}$. The parameters $\left(\tau_{i}, I_{i}\right)$ of the distributions are extracted by computer analyses.

The principle of an angular correlation measurement is shown in Figure 2. The angle between the two $0.5 \mathrm{I} \mathrm{I} \mathrm{MeV} \mathrm{photons} \mathrm{emitted} \mathrm{in} \mathrm{the} 2 \gamma$ annihilation process is $180^{\circ}$ if the electron-positron system is at rest. A deviation from $180^{\circ}$ occurs if the two particles have a net momentum at the annihilation. The angular correlation set-up determines the number of $2 \gamma$ annihilations as a function of the angle $\theta$, the deviation from $180^{\circ}$. A typical angular correlation curve for a metal is shown in Figure 2. The parabolic component is due to annihilation with the valence electrons. The normally-used long-slit geometry of effective detector areas determines the distribution of one component $k_{z}$ of the sum of the momenta of the two photons $\mathbf{k}=\mathbf{k}_{1}+\mathbf{k}_{2}$ (see below).

In ice, roughly $74 \%$ of the positrons form Ps, of which $\frac{1}{4}$ are para-Ps and $\frac{3}{4}$ are ortho-Ps. In para-Ps, the particles have opposite spin directions. It can annihilate intrinsically into two photons with a lifetime of 0.13 ns. If para-Ps is localized on annihilation the para-Ps angular correlation contribution is determined by the momentum distribution in its zero-point motion (see below). This para-Ps contribution is normally called the narrow component. Its full width at half maximum (fwhm) is normally 2-4 mrad. Delocalized para-Ps in a solid, i.e. para-Ps in a Bloch-function state, gives rise to several very narrow peaks in the angular correlation curves, as discussed in more detail below (see Figs 3 and 4). Ortho-Ps in vacuum annihilates into three photons with a lifetime of r $40 \mathrm{~ns}$. Two-photon annihilation is not
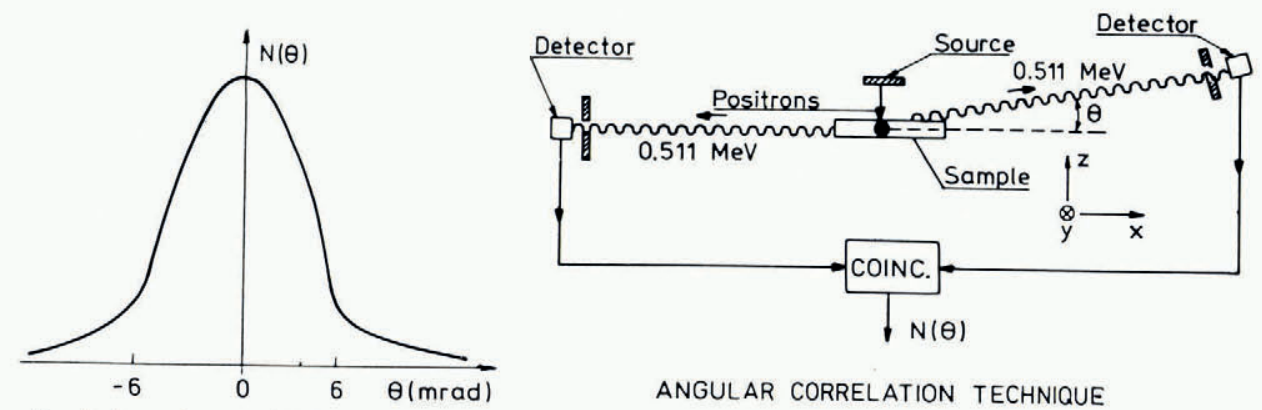

ANGULAR CORRELATION TECHNIQUE

Fig. 2. A typical angular correlation curve for a metal and a diagram of the experimental set-up for angular correlation measure-
ments. 
possible if the positron and electron have the same spin directions as in ortho-Ps. However, the positron in ortho-Ps can annihilate into two photons with an electron with opposite spin during Ps-molecule collisions in condensed matter. This pick-off annihilation (lifetime I-5 ns) is the dominating decay mode for ortho-Ps in liquids and solids. The free positrons (i.e. the positrons not forming Ps) annihilate with the outer electrons of the molecules into two photons with a typical lifetime of $0.4 \mathrm{~ns}$. Because the binding energy of Ps is only $6.8 \mathrm{eV}$, a thermalized positron cannot pick-off an electron bound in the molecules to form Ps in normal solids. As both the free positron and the Ps pick-off annihilations occur with the outer electrons in the molecules, the angular correlation contributions are broader (fwhm $=8-$ I I mrad) than that of the para-Ps contribution ( $\mathrm{fwhm}=2-4 \mathrm{mrad}$ for localized, and very narrow for delocalized, para-Ps).

\section{Theory}

We shall now discuss the theoretical expressions for the angular correlation curves and lifetimes in some detail. The expressions are used in the next subsection where we compare the information that can be obtained for the Ps states with that obtainable for the similar electron and positron states. The formulae are calculated within quantum electrodynamics in the non-relativistic approximation (see Mogensen (unpublished), Mogensen and Eldrup ( $1977[\mathrm{~b}])$ for a more detailed discussion).

To a fairly good approximation we may assume that the state of a system consisting of a Ps atom and $n-\mathrm{I}$ electrons is described by the non-relativistic wave function

$$
\psi=c_{\mathrm{I}} A\left\{\psi_{\mathrm{P}}\left(\mathbf{x}_{\mathrm{I}}, \mathbf{x}_{\mathrm{p}}\right) \psi_{1}\left(\mathbf{x}_{2}, \ldots, \mathbf{x}_{n}\right)\right\},
$$

where $c_{\mathrm{I}}$ is a normalization constant $\left(c_{\mathrm{I}}=n^{\frac{1}{2}}\right.$, if the Ps-electron does not overlap the other electrons), and $A$ is the antisymmetry operator. $\psi_{\mathrm{P}}\left(\mathbf{x}_{\mathrm{I}}, \mathbf{x}_{\mathrm{p}}\right)$ is the Ps wave function and $\psi_{\mathrm{I}}\left(\mathbf{x}_{2}, \ldots, \mathbf{x}_{n}\right)$ is the antisymmetric wave function for the $n-\mathbf{I}$ electrons $\left(\mathbf{x}_{j}=\mathbf{r}_{j} \sigma_{j}\right.$,

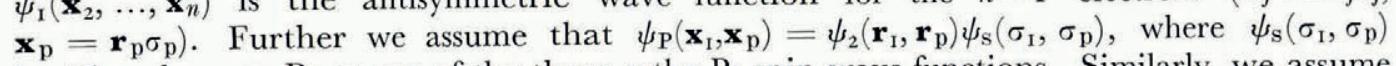
is either the para-Ps or one of the three ortho-Ps spin wave functions. Similarly, we assume that $\psi_{\mathrm{I}}\left(\mathbf{x}_{2}, \ldots, \mathbf{x}_{n}\right)=\psi_{3}\left(\mathbf{r}_{2}, \ldots, \mathbf{r}_{n}\right) \psi_{4}\left(\sigma_{2}, \ldots, \sigma_{n}\right)$, and that the state of the $n-\mathrm{I}$ spins can be described by a uniform ensemble (i.e. we assume equal probability and random phase).

The calculation results in the following spin-averaged probability per unit time for annihilation into two photons, of which one is emitted into the space angle $\mathrm{d} \Omega$, and for which the sum of the wave vectors $\mathbf{k}=\mathbf{k}_{\mathrm{r}}+\mathbf{k}_{2}$ is within $\mathrm{d} \mathbf{k}$

$$
\begin{aligned}
\mathrm{d} w= & \frac{r_{0}{ }^{2}}{(2 \pi)^{3}} \mathrm{~d} \mathbf{k} \mathrm{d} \Omega \frac{\left|c_{\mathrm{I}}\right|^{2}}{n}\left[\left\{2\left|\int \mathrm{d} \mathbf{r}_{\mathrm{I}} \exp \left(-\mathrm{i} \mathbf{k} \cdot \mathbf{r}_{\mathrm{I}}\right) \psi_{2}\left(\mathbf{r}_{\mathrm{I}}, \mathbf{r}_{\mathrm{I}}\right)\right|^{2}\right\}_{\text {para }}+\right. \\
& \left.+\frac{n-\mathrm{I}}{2} \int \mathrm{d} \mathbf{r}_{\mathrm{I}} \ldots \int \mathrm{d} \mathbf{r}_{n-\mathrm{I}}\left|\int \mathrm{d} \mathbf{r}_{n} \exp \left(-\mathrm{i} \mathbf{k} \cdot \mathbf{r}_{n}\right) \psi_{2}\left(\mathbf{r}_{\mathrm{I}}, \mathbf{r}_{n}\right) \psi_{3}\left(\mathbf{r}_{2}, \ldots, \mathbf{r}_{n}\right)\right|^{2}\right],
\end{aligned}
$$

where the subscript para indicates that the term is only different from zero if $\psi_{\mathrm{s}}\left(\sigma_{\mathrm{I}}, \sigma_{\mathrm{p}}\right)$ is equal to the para-Ps spin function. We use units in which $\hbar=c=\mathrm{I}$ and then $r_{\mathrm{o}}=\alpha / m_{\mathrm{e}}$ where $\alpha$ is the fine structure constant and $m_{\mathrm{e}}$ the electron mass. The standard linear-slit angular-correlation set-up determines to a good approximation the distribution obtained by integrating over $k_{x}, k_{y}$, and $\Omega$ in Equation (2).

The total $2 \gamma$ annihilation probability (i.e. the reciprocal lifetime) is obtained by an integration over $\mathbf{k}$ and $\Omega$. We get

$$
\begin{aligned}
w=2 \pi r_{0}{ }^{2} \frac{\left|c_{\mathrm{I}}\right|^{2}}{n}\left[\left\{2 \int \mathrm{d} \mathbf{r}_{\mathrm{I}}\left|\psi_{2}\left(\mathbf{r}_{\mathrm{I}}, \mathbf{r}_{\mathrm{I}}\right)\right|^{2}\right\}_{\text {para }}+\right. & \\
& \left.+\frac{n-\mathrm{I}}{2} \int \mathrm{d} \mathbf{r}_{\mathrm{1}} \ldots \int \mathrm{d} \mathbf{r}_{n}\left|\psi_{2}\left(\mathbf{r}_{\mathrm{I}}, \mathbf{r}_{n}\right)\right|{ }^{2}\left|\psi_{3}\left(\mathbf{r}_{2}, \ldots, \mathbf{r}_{n}\right)\right|^{2}\right] .
\end{aligned}
$$


In Equations (2) and (3) we have neglected two "exchange" terms which are zero if the Ps electron does not overlap the electrons described by $\psi_{3}\left(\mathbf{r}_{2}, \ldots, \mathbf{r}_{n}\right)$, i.e. we neglect the influence of the antisymmetry operator in Equation ( $\mathrm{I}$ ). In normal cases these two terms are probably small compared to the two terms shown. A completely satisfactory derivation of Equations (2) and (3) has probably not yet been given. However, they seem to be the only available general expressions. They reduce to well-known expressions in simple cases like the independent and free-particle approximations, and the para-Ps intrinsic annihilation. Hence they are probably correct.

The first term in Equations (2) and (3) is the para-Ps intrinsic annihilation probability, while the second term gives the pick-off annihilation probability. If we assume the following Ps space-coordinate wave function

$$
\psi_{2}\left(\mathbf{r}_{1}, \mathbf{r}_{\mathrm{p}}\right)=\psi_{\mathrm{c}}(\mathbf{r}) f(\mathbf{R}),
$$

i.e. a product of a function $\psi_{\mathbf{c}}(\mathbf{r})$ of the centre-of-mass coordinate $\mathbf{r}=\left(\mathbf{r}_{\mathrm{I}}+\mathbf{r}_{\mathrm{p}}\right) / 2$ and a function $f(\mathbf{R})$ of the relative coordinate $\mathbf{R}=\mathbf{r}_{\mathrm{I}}-\mathbf{r}_{\mathrm{p}}$ (e.g. the s-state wave function), we find from the first term in Equation (2)

$$
\mathrm{d} w \propto\left|\int \mathrm{d} \mathbf{r} \exp (-i \mathbf{k} \cdot \mathbf{r}) \psi_{\mathrm{c}}(\mathbf{r})\right|^{2} \mathrm{~d} \mathbf{k} \mathrm{d} \Omega .
$$

Hence the angular correlation curve for the intrinsic para-Ps annihilation is determined by the numerical value of the Fourier transform of the Ps centre-of-mass wave function.

In the free positron case, the spin-averaged total $2 \gamma$ annihilation probability is

$$
w=\pi r_{0}^{2} \sum_{i=1}^{n} \int \mathrm{d} \mathbf{r}_{1}, \ldots \int \mathrm{d} \mathbf{r}_{n}\left|\psi\left(\mathbf{r}_{1}, \ldots, \mathbf{r}_{n}, \mathbf{r}_{i}\right)\right|^{2},
$$

where $\psi\left(\mathbf{r}_{1}, \ldots, \mathbf{r}_{n}, \mathbf{r}_{\mathrm{p}}\right)$ is the non-relativistic wave function for the space variables of the $n$ electrons and one positron. Hence, $w\left(=\tau^{-1}\right.$, where $\tau$ is the lifetime) is proportional to the density of electrons at the positron, as in the pick-off term in Equation (3).

If we assume that $\psi_{\mathrm{c}}(\mathbf{r})$ in Equation (5) is a Bloch function, i.e. that

$$
\psi_{\mathrm{c}}(\mathbf{r})=\exp (i \mathbf{p} \cdot \mathbf{r}) \sum_{\mathbf{g}} a_{\mathbf{g}} \exp (i \mathbf{g} \cdot \mathbf{r}),
$$

where $\mathbf{p}$ is the crystal wave vector and $\mathbf{g}$ a vector in the reciprocal lattice, we obtain the intrinsic para-Ps contribution

$$
\mathrm{d} w \propto \sum_{\mathbf{g}}\left|a_{\mathbf{g}}\right|^{2} \delta(\mathbf{p}-\mathbf{k}+\mathbf{g}) \mathrm{d} \mathbf{k} \mathrm{d} \Omega .
$$

As discussed by Mogensen and others (1971), this explains the presence of the side peaks at angles $\theta=\hbar g_{z} / m_{\mathrm{e}} c$ in the angular correlation curves of ice (see Figs 3 and 4 ), where $g_{z}$ is the projection of $\mathbf{g}$ in the $z$-direction of the set-up.

If $P s$ is localized in a defect, the para-Ps intrinsic annihilation component of the angular correlation curve is given by the Fourier transform of the zero-point-energy wave function. For example, if we assume that $\psi_{\mathrm{c}}(\mathbf{r})$ in Equation (5) is a harmonic-potential wave function of lowest energy $E_{0}$, we get a Gaussian linear-slit angular correlation curve of full width at half maximum (fwhm) $\Gamma_{\theta}$, for which

$$
\Gamma_{\theta} \Gamma_{z}=10.7 \operatorname{mrad} \AA \text {, }
$$

where $\Gamma_{z}$ is the fwhm of the probability distribution of the $z$ component of the centre-of-mass coordinate $\mathbf{r}$. Hence, the fwhm of the narrow component is inversely proportional to the width of the probability distribution, i.e. it gives a rough measure of the size of the defect in which para-Ps is trapped. 


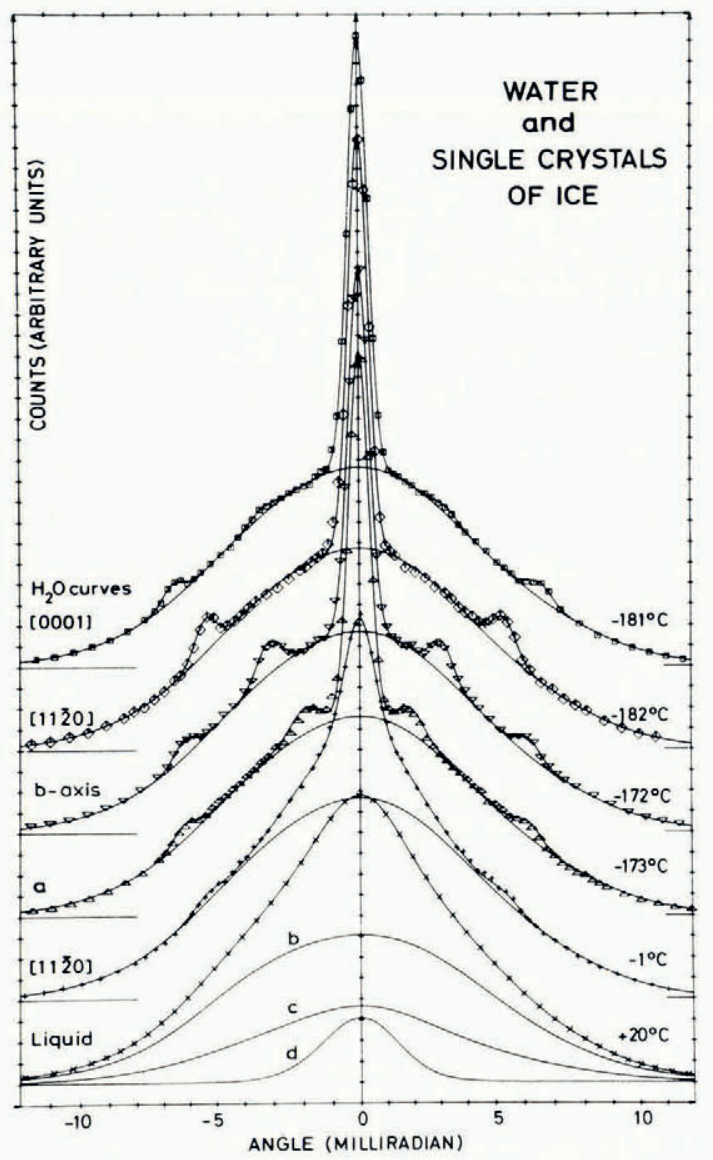

Fig. 3 .

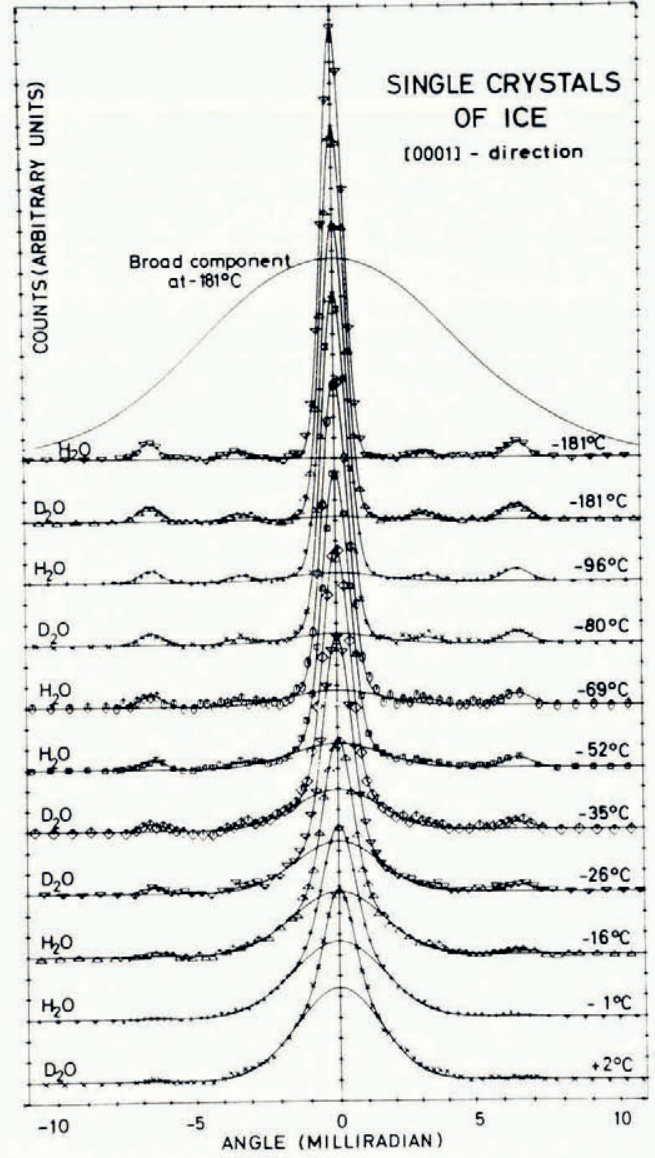

Fig. 4 .

Fig. 3. The measured points and the fitted curves of the angular correlation distributions in water and single crystals of ice. The $z$-direction of the b-axis curve is perpendicular to the c-axis and tilted $30^{\circ}$ from the a-axis. The $z$-direction of curve $a$ is in the plane of the $a$ - and c-axes and tilted $20.6^{\circ}$ from the c-axis. Curves $b, c$, and d are the "free" positron, ortho-Ps pick-off, and para-Ps contributions to the water curve, respectively. The measured curves are normalized to equal area. The uncertainties are smaller than or equal to the size of the points.

Fig. 4. The temperature dependence of the angular correlation curves for single crystals of $\mathrm{H}_{2} \mathrm{O}$ - and $\mathrm{D}_{2} \mathrm{O}$-ice oriented along the $c$-axis. The measured points and the fitting curves are shown after the subtraction of the broad component from the normalized curves. Only uncertainties greater than the size of the points are shown.

\section{Measurable properties}

An important method of studying defects, such as point defects and their agglomerates, in insulators and semiconductors is to study electrons on the defects by use of e.g. optical absorption or EPR measurements (Crawford and Slifkin, 1972-75; Stoneham, 1975; Henderson and Hughes, 1976). More detailed information on defects is available for a semiconductor, silicon, than for any other system, mainly because EPR experiments on the defects (free radicals, trapped electrons) have been systematically carried out yielding detailed defect models. Similarly, very detailed information on the structures of colour centres, etc. in ionic crystals has been obtained by use of EPR and optical measurements. Results of optical absorption measurements on electrons trapped in various defects in pure ice have recently been published (Buxton and others, 1975, 1977; Kawabata, 1976), while the influence of 
defects on the conductivity of mobile electrons in ice has been studied by J. M. Warman and J. B. Verberne (private communication in 1977). Because of the very important role played by electrons in the defect studies of insulators (a fact which is perhaps not fully realized in ice research), a short discussion of the type of information that can be obtained in the cases of the electron (whether mobile, trapped in defects, or the unpaired electron on a free radical), and Ps atom (mobile or trapped) will be helpful for the understanding of the discussion of the Ps trapping in the next section.

In principle, measurements might give various types of information on the states of the three light particles, as illustrated by the following remarks:

(I) The wave function $\psi$ itself cannot be determined in a single experiment. This is a general property of quantum mechanics.

(2) The probability distribution $|\psi|^{2}$ of a light particle in real space can apparently not be measured in any detail for any of the light particles.

(3) The numerical value of the Fourier transform of $\psi$ (measurable for para-Ps) is similar to the real-space distribution $|\psi|^{2}$ with respect to the information content of the data.

(4) For a bound particle (trapped on a defect or impurity; unpaired electron on a free radical), properties that essentially depend only on the ground-state wave function might be measurable.

(5) For a bound particle, other measurable properties (e.g. optical absorption) depend on both the ground state and the excited state(s).

(6) Properties that can be expressed by single numbers (e.g. trapping rates, mobilities, etc., for mobile particles) might be measurable for trapped and mobile particles.

The main reasons for the importance of EPR measurements on free radicals and electrons trapped in defects are that the measured electron properties are essentially only dependent on the ground-state wave function (case (4)), and that an EPR measurement, being a resonance technique, gives results which are very dependent on the details of the electrondefect structure. However, optical absorption results for electrons trapped on defects depend on both the ground and the excited state(s) and, hence, several theoretical models of electrondefect structure can normally fit the measured absorption spectra. Roughly speaking, the only measurable properties of mobile electrons (conduction-band or excess electrons) seem to be single-number properties (which might depend on the orientation of the crystals), such as mobilities, Hall coefficients, and trapping rates. For Ps atoms (trapped or mobile), the numerical value of the Fourier transform of the wave function can be determined (case (3)). Such detailed information on the state is not available for the other two particles-the electron and the positron - in any system. However, the EPR spectra, etc., of electrons on defects give more information on the electron-defect system than in the trapped Ps case, mainly because the EPR spectra are better defined experimentally and probably easier to interpret than the narrow component of the angular correlation curves (see below). On the other hand, much more information is available on the mobile Ps atom (case (3)) than is available for the mobile electron (case (6)). In this context it must be realized that Ps trapping at two different times (the para-Ps and the ortho-Ps lifetimes) can be measured (trapping rate determination, case (6)). For free positrons trapped in defects the Fourier transform of the positron-electron wave-function overlap is determined in an angular correlation measurement. As this is a ground-state property, more detailed information is probably available on the localized positrons than on the localized electrons in the fairly frequent cases where the EPR measurements on the electrons do not give very valuable information. For example, optical absorption studies of solvated electrons do not seem to give as valuable information as angular correlation studies of solvated positrons.

In the case of positrons trapped in vacancies and clusters of vacancies in metals, the available information on the positron-many-electron state, corresponds to case (4) (angular 
correlation) and case (6) (trapping rate, lifetime (Equation (6))), while only the scattering properties of the defects can be measured for the mobile electron in a metal (case (6)). Hence, the positron trapping method for metals is fairly similar to F-centre spectroscopy in ionic crystal work. This fact explains the usefulness of the PAT method in metal defect research.

\section{RESUlts AND DISGUSSION}

In this section we shall discuss our results and their interpretation in terms of the trapping of Ps in vacancies. Obviously, we cannot discuss the interpretation in detail here. Hence, the discussion will be fairly qualitative, and we refer to the series of papers on positron annihilation in ice (see the Introduction) for a more complete discussion. In particular, the effects of temperature on the properties of Ps and the interpretation in terms of Ps trapping in vacancies in ice is discussed in great detail by Mogensen and Eldrup (1977[b]).

\section{Results}

For the angular correlation measurements, a standard linear-slit set-up was used (West, 1973). The detectors covered a solid angle of $0.48 \times 178 \mathrm{mrad}$ as seen from the sample. We used an external ${ }^{22} \mathrm{NaCl}$ positron source. The angular correlation curves were analysed by use of the least-squares fitting programme Paacfit (Kirkegaard and Mogensen, 1973). In the work on pure ice we measured angular correlation curves for monocrystals of light and heavy ice for various crystal orientations and as a function of temperature from $-182^{\circ} \mathrm{C}$ to the melting point, a total of about $5^{\circ}$ curves. Some of the measured curves are shown in Figures 3 and 4 , in Figure 4 with a broad component subtracted to show more clearly the narrow ( $\approx$ I mrad) components.

The narrow central and side peaks are caused by the intrinsic annihilation of para-Ps. As discussed above (Equation (8)), there are sharp side peaks if the para-Ps centre-of-mass wave function is a Bloch function. The side peaks in Figure 3 are found at the expected angles calculated by use of the lattice parameters of ice. The broad component of the curve is mainly caused by pick-off annihilation of ortho-Ps and free-positron annihilation. With increasing temperatures the intensities of the narrow peaks decrease followed by a simultaneous increase in intensity of a "middle-broad" ( $\approx 3.8 \mathrm{mrad}$ ) component as shown in Figure 4. This $3.8 \mathrm{mrad}$ component is caused by intrinsic annihilation of para-Ps localized on the vacancies. Except for a small correlation due to the change in the pick-off annihilation probability of para-Ps on trapping, the intensity and shape of the broad component are independent of temperature. Hence, the main information gained by use of the angular correlation method is the change in the para-Ps centre-of-mass wave function with temperature as shown in Figure 4. The temperature at which the intensity of the central peak attained a certain value was roughly $3.8 \mathrm{deg}$ higher for $\mathrm{D}_{2} \mathrm{O}$ ice than for $\mathrm{H}_{2} \mathrm{O}$ ice. This correlates well with the fact that the melting point of $\mathrm{D}_{2} \mathrm{O}$ ice is $3.8 \mathrm{deg}$ higher than that of $\mathrm{H}_{2} \mathrm{O}$ ice. Figure 5 shows the intensity of the $3.8 \mathrm{mrad}$ component versus temperature.

The lifetime spectrometer used was a conventional fast-slow coincidence system (West, 1973) with a time resolution characterized by a fwhm $=0.42 \mathrm{~ns}$. The source used was about $6 \mu \mathrm{Ci}$ of ${ }^{22} \mathrm{NaCl}$ sealed between two $\mathrm{I} \mathrm{mg} / \mathrm{cm}^{2}$ nickel foils. The fraction of positrons annihilating in the source and foils was estimated at $10 \%$. The lifetime spectra were analysed by use of the least-squares fitting programme Positronfit (Kirkegaard and Eldrup, 1974).

Results of a detailed lifetime study of pure ice at temperatures above $-180^{\circ} \mathrm{C}$ were previously published by Eldrup and others (1972). We have recently measured some new high-quality lifetime spectra for light ice between $-175^{\circ} \mathrm{C}$ and the melting point (Mogensen and Eldrup, I977[b]). The lifetime curves consisted of two short-lived components caused by para-Ps intrinsic $\left(\tau_{1} \approx 0.12 \mathrm{~ns}\right)$ and free positron $\left(\tau_{2} \approx 0.4 \mathrm{~ns}\right)$ annihilation and one or more 


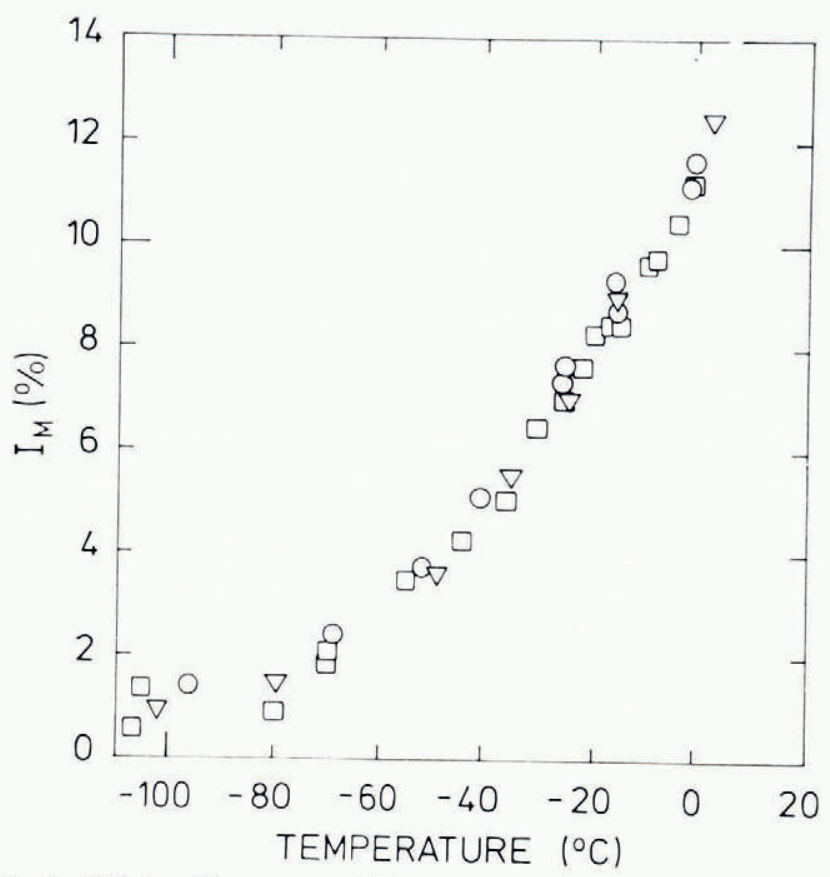

Fig. 5. The intensity of the "middle-broad" component (fwhm $=3.94,3.70$, and 3.42 mrad for $\mathrm{H}_{2} \mathrm{O}$ a-axis, $\mathrm{H}_{2} \mathrm{O}$ c-axis, and $\mathrm{D}_{2} \mathrm{O}$ c-axis, respectively) versus temperature. The squares and circles are for $\mathrm{H}_{2} \mathrm{O}$ a- and c-axis crystals, respectively, and the triangles for $\mathrm{D}_{2} \mathrm{O}$ c-axis crystals.

long-lived components caused by ortho-Ps pick-off annihilation. Only the latter component(s) could be clearly resolved. The intensities and lifetimes of the long-lived components were therefore the main information obtained by use of the lifetime method. At low temperatures $\left(<c .-100^{\circ} \mathrm{C}\right)$ one long lifetime of $\tau_{3} \approx 0.7 \mathrm{~ns}$ was found. The long lifetime increased with temperature to about $\mathrm{I}$ ns at the melting point, $I_{3}$ being essentially constant at $5 \mathrm{I} \pm 3 \%$. A new component of constant lifetime $\tau_{4}=\mathrm{I} .7 \mathrm{~ns}$ was found, which had an intensity that was close to $0.5 \%$ at $-100^{\circ} \mathrm{C}$, increased to $\mathrm{I} .5 \%$ at $-40^{\circ} \mathrm{C}$ and then sharply increased to $5.1 \%$ at $-5^{\circ} \mathrm{C}$. We interpret the change in $\tau_{3}$ with temperature as due to the influence of monovacancies on the probability of ortho-Ps pick-off annihilation. The $\tau_{4}, I_{4}$ component is tentatively interpreted as caused by the influence of divacancies on the ortho-Ps pick-off process.

Why Ps trapping in vacancies?

From the point of view of ice physics the most important result of our work is the information obtained on vacancies. Hence, we shall here discuss in some detail why, in particular, the vacancy is thought to be the cause of the measured changes in the lifetime spectra and the angular correlation curves. The reason we did not consider seriously the Ps trapping in vacancies in our earlier studies (1969-72, reviewed by Mogensen and Eldrup (1973)) was simply that the relative vacancy concentration in ice was stated to be very low in the literature $\left(\approx \mathrm{IO}^{-10}\right.$ vacancies per ice molecule at $-\mathrm{I}^{\circ} \mathrm{C}$ ). Such a low concentration cannot influence Ps. As Ps is trapped in hole-type defects in a solid, an alternative defect to consider was the L-defect. However, the data accumulated by the year I 973 showed fairly clearly that Ps was probably not trapped in L-defects. The measured effects associated with Ps trapping seemed 
not to vary with the L-defect concentration in a reasonable way, as far as the L-defect concentration (with or without an added positive ion-state defect) was thought to be known. Hence, we had to conclude that Ps was either trapped in some unknown defect or in a vacancy, the vacancy concentration being roughly $\mathrm{I}^{4}$ times higher than normally assumed. Today we strongly believe that the latter possibility is correct for the following reasons.

First, our results (Eldrup, 1976; Eldrup and others, 1978) for irradiated ice showed that Ps is trapped in a defect that seems to be identical (within the uncertainty) to the defects which trap Ps in pure ice at high temperatures and in HF-doped ice at all temperatures. Radiation chemists strongly believe they have found interstitial $\mathrm{OH}$ radicals which are created by the $\gamma$-irradiation, at concentrations of roughly $10^{-6}$ per water molecule (p.p.m.) at the irradiation dose we used. Hence, we expected to have roughly p.p.m. concentrations of vacancies in our irradiated samples. Very probably, Ps is trapped in these vacancies.

Secondly, the Ps-trapping defect in irradiated and in rapidly cooled HF-doped ice annealed in just the way expected of a vacancy annealing. The annealing resulted in very long ortho-Ps lifetimes that could be interpreted as caused by Ps trapping in divacancies and polyvacancies, which, of course, must be expected to form when vacancies begin to migrate. Similar effects of the annealing of vacancies in metals have recently been found by the positron-trapping method (Eldrup and others, 1976).

Thirdly, if self-diffusion in ice takes place by a vacancy mechanism, which seems to be generally accepted, the value of the diffusion constant $D$ leads to a lower limit to the vacancy concentration (Mogensen and Eldrup, I977[a]). The average jump frequency of the molecules can be calculated from $D$. Only the four molecules neighbouring the vacancy can jump at a certain time with a frequency somewhat smaller than a typical phonon frequency. This puts a lower limit to the vacancy concentration which appears to be a few p.p.m. at the melting point. Of course, this estimate is independent of the Ps trapping results. A vacancy concentration at the p.p.m. level can be expected to trap Ps.

Fourthly, several other facts speak strongly in favour of Ps trapping in vacancies. For example, the sum of the energies of formation and migration of vacancies derived from our results (see below) is roughly equal to the activation energy of diffusion, as expected. In general, the model of Ps trapping in vacancies can explain our data.

It is important to realize that the estimated value of the vacancy concentration in ice found in the literature is very probably much too low. The formation energy of water molecule vacancies $E_{\mathrm{f}}$ has been estimated to be about $0.5 \mathrm{eV}$. This value has been obtained on the assumption that it should be close to the sublimation energy (Hobbs, 1974; Fletcher, 1970). With the relative vacancy concentration $c$ given by

$$
c=\exp \left(S_{\mathrm{f}} / k\right) \exp \left(-E_{\mathrm{f}} / k T\right)
$$

where $S_{\mathrm{f}}$ is the vacancy formation entropy, $k$ Boltzmanns constant, and $T$ the temperature, and on the assumption that the entropy factor is approximately unity, $c$ becomes about $\mathrm{IO}^{-10}$ close to the melting point for $E_{\mathrm{f}}=0.5 \mathrm{eV}$. However, this estimate of $E_{\mathrm{f}}$ probably leads to too large a value for $E_{\mathrm{f}}$ and hence to much too low a value for $c$. In the rare gas solids (metals), the formation energies of a vacancy amount to roughly $70 \%(30 \%)$ of the sublimation energies.

To summarize, we may conclude that many independent arguments point towards Ps trapping in vacancies in ice.

\section{Vacancy formation energy}

By use of the pure-ice results shown in Figures $3^{-5}$ a value of the vacancy formation energy $E_{\mathrm{f}}$ can be estimated. However, this estimate of $E_{\mathrm{f}}$ proved to be rather difficult. A simple, "classical" model of the localization of Ps in vacancies is the so-called trapping model (West, 1973). The main assumptions used in this model are (I) Ps annihilates either from a 
delocalized or from a localized state, (2) Ps is formed in the delocalized state, and (3) Ps is trapped with a certain trapping probability per unit time, which is proportional to the vacancy concentration. Hence, it is assumed that trapping competes in a simple way with pick-off annihilation $\left(\tau_{3}=0.7 \mathrm{~ns}\right)$ and intrinsic annihilation $\left(\tau_{1}=0.12 \mathrm{~ns}\right)$ for ortho-Ps and para-Ps, respectively. Of course, this model predicts that more ortho-Ps than para-Ps atoms are trapped at a certain defect concentration, simply because ortho-Ps lives roughly six times longer than para-Ps. However, our experimental results showed that ortho-Ps and para-Ps seem to be trapped to about the same degree at a certain vacancy concentration, i.e. at a certain temperature. Hence, the simple trapping model cannot explain the results. This is the main difficulty in the estimate of $E_{\mathrm{f}}$. Several other more complicated models were applied in the interpretation of the data. An extended trapping model, which included the presence of divacancies and a probability of detrapping, fitted the data fairly well, but the fitting parameters were somewhat unrealistic. In an alternative, more "quantum-mechanical" model it was assumed that the Ps centre-of-mass wave function is a linear combination of a Bloch function and a function localized on the vacancies. This wave function was assumed to be established in a time which is short compared with the para-Ps lifetime of o. 2 ns. This "linear-combination" model did not explain the data in detail either. Of course, more elaborate models with many fitting parameters can fit the measured data by a suitable choice of the parameters. Altogether the analysis of the present experimental data on Ps trapping in temperature-created vacancies showed that no simple model could convincingly account for the observations. Thus no detailed quantitative correlation can be obtained between the amount of Ps trapping and the vacancy concentrations. On the other hand, the "trapping model with escape" suggested that $E_{\mathrm{f}} \approx 0.2 \mathrm{eV}$, and using the linear-combination model on the lifetime and angular correlation results separately gives values for $E_{\mathrm{f}}$ of $0.13-0.18 \mathrm{eV}$. In this context it must be realized that the dependence of the vacancy concentration on temperature might be more complicated than that described by Equation (I0), because of the very probable existence of bound states between the vacancy and some of the hydrogenbond defects (see below).

A rough way to estimate $E_{\mathrm{f}}$ from the present measurements is to note that trapping of Ps sets in above about $-100^{\circ} \mathrm{C}$ (Figs 4 and 5). Previous results in HF-doped ice (Eldrup and others, 1972; Mogensen and Eldrup, 1973) showed that Ps trapping sets in at a relative defect concentration of $\mathrm{IO}^{-8}-\mathrm{IO}^{-6}$ (similar to positron trapping in vacancies in metals that sets in at a concentration of roughly $\mathrm{IO}^{-7}$ ). Using Zener's approximation (e.g. Ramseier, 1967) for $S_{\mathrm{f}}$ in Equation (10), we get $0.26 \mathrm{eV}<E_{\mathrm{f}}<0.35 \mathrm{eV}$. (For $\exp \left(S_{\mathrm{f}} / k\right) \approx \mathrm{I}$, $0.20 \mathrm{eV}<E_{\mathrm{f}}<0.28 \mathrm{eV}$.)

From the above discussion we can conclude that, irrespective of the model used in the analysis of the present data on Ps trapping in temperature-created vacancies, $E_{\mathrm{f}}$ was roughly 0.2 to $0.35 \mathrm{eV}$, which is much lower than the normally used value of $0.5 \mathrm{eV}$. This is in agreement with our results from the Ps trapping studies of the annealing behaviour of irradiated and HF-doped ice (Eldrup, I976; Eldrup and others, 1978). A main conclusion was that the vacancy migration energy $E_{\mathrm{m}}=0.34 \pm 0.07 \mathrm{eV}$, and hence, $E_{\mathrm{f}}=0.28 \pm 0.07 \mathrm{eV}$, because $E_{\mathrm{f}}+E_{\mathrm{m}}=Q$, the activation energy for diffusion that seems well determined at o.62 eV (Ramseier, 1967).

\section{Vacancy bound states with other defects}

From the above discussion we can conclude that several independent experimental facts lead to a vacancy concentration at the melting point of roughly I-IO p.p.m., i.e. more than $\mathrm{IO}^{4}$ times higher than the previously assumed value of $1^{-10}$ (Hobbs, I974; Fletcher, 1970). In the "hydrogen-bond-defects" theory of the electric properties of ice (Hobbs, I974; Fletcher, 1970; Whalley and others, I973), the main role is played by the defects on the 
hydrogen bonds, the ion-state defects $\mathrm{H}_{3} \mathrm{O}^{+}$and $\mathrm{OH}^{-}$and the Bjerrum, orientational L- and D-defects. The presence of vacancies has largely been neglected in the theory. The fact that the vacancy concentration is comparable to that of the hydrogen-bond defects in a large temperature interval will probably enforce a pronounced change in this theory. The bound states of vacancies and some of the other four defects may be stable (see e.g. Seidensticker and Longini, I969). For instance, the question of the existence and possible configuration of D-defects in ice has been a matter of extensive discussion, since the simple picture of two protons on a linear hydrogen bond is unrealistic because of its high energy. If the surroundings of the D-defect are allowed to relax, this energy is considerably reduced. However, it seems that the space available in a vacancy might make a D-defect-vacancy aggregate energetically favourable. Thus, a high vacancy concentration may entail that almost all D-defects are bound to vacancies. Also some impurity-vacancy bound states might be stable. In particular, the vacancy bound states with $\mathrm{HF}$ and $\mathrm{NH}_{3}$ and/or dissociation products might be of importance in relation to the dielectric properties of $\mathrm{HF}$ - or $\mathrm{NH}_{3}$-doped ice. Hence, the equilibrium concentrations and transport properties of the four hydrogen-bond defects in pure and doped ice probably cannot be calculated without taking into account the presence of vacancies. Clearly, the necessary inclusion of the vacancies in the "hydrogen-bond-defects" theory of the dielectric properties of ice makes the theory more complicated. Several new parameters, such as the vacancy concentration and the vacancy hydrogen-bond defect binding energies, must probably be introduced into the new theory, which may then contain so many parameters that practically any experimental result could be fitted by variation of the parameters. On the other hand, some of the new parameters such as $E_{\mathrm{f}}$ and the vacancy concentration are now available through experimental studies of Ps trapping. Maybe electron trapping in vacancies will give information on vacancy parameters in the future.

Of course the high vacancy concentration might also influence other ice properties. For example, the presence of substitutional HCl in ice (as indicated by the measurements of Huber and others (1973)) is difficult to understand because the chloride ion is significantly larger than the oxygen ion. However, this might be explained if we assume that one or more vacancies are bound to $\mathrm{HCl}$ thereby relaxing the strain due to the presence of HCl. Similarly, we might expect that other large impurities in substitutional positions can be stable due to bound state formation with vacancies. The smaller impurities like $\mathrm{H}$ (created by irradiation) and $\mathrm{He}$ might also be expected to get trapped in vacancies, although they apparently are also found in interstitial positions in ice.

\section{Additional remarks}

As mentioned in the Introduction, only the vacancy aspects of our pure-ice work are discussed in detail in this paper. However, some short remarks on other aspects of the work may be appropriate here.

Angular correlation work at Queen's University, Kingston, Canada (a paper on this is in preparation by M. Eldrup, R. J. Douglas, and L. Lupton) has shown that Ps is essentially thermalized at the time of annihilation even at liquid-helium temperature, a result which is very important for the understanding of Ps physics in general.

The para-Ps component of the angular correlation curve is given by the numerical value of the Fourier transform of the Ps centre-of-mass wave function. Such detailed information on wave functions is not available for the other two light particles, the electron and the positron. Our results give direct information on the wave function itself. For example, an independentparticle model of the influence of the thermal motion of the molecules on the Ps Bloch function predicts that the ratio of a side-peak intensity to the central-peak intensity (see Fig. 4) decreases proportionally to the square of the corresponding Debye-Waller factor. However, a much stronger decrease of the side-peak intensities was measured. 
Generally speaking, the theory of the trapping of a particle in a solid or liquid, i.e. the change from a mainly delocalized to a mainly localized wave function by simultaneous loss of energy to phonons, etc., is not well developed. Our Ps trapping results for ice may be valuable in a test of such theories, because of the unusually large amount of information that can be experimentally obtained as a result of the particular characteristics of the annihilation process, as discussed above. Maybe the difficulties encountered in the detailed interpretation of the influence of vacancies on the Ps state in ice will also be encountered in electron and positron trapping studies, when details of the trapping process, similar to those we measured for Ps, can be obtained for such cases in the future.

\section{Conclusion}

The behaviour of Ps in single crystals of pure light and heavy ice has been studied as a function of temperature. Several independent experimental results strongly indicate that Ps is trapped in vacancies at higher temperatures. The vacancy concentration is at the p.p.m. level at the melting point, i.e. roughly $1^{4}$ times higher than previously assumed. This high vacancy concentration can also be estimated using self-diffusion results for ice. Because we could not determine the exact nature of the influence of the vacancies on Ps behaviour, it was difficult to derive a very well-defined vacancy formation energy $E_{\mathrm{f}}$ from the temperature variations. $E_{\mathrm{f}}$ was derived to be roughly $0.2-0.35 \mathrm{eV}$ in agreement with the result $E_{\mathrm{f}}=$ $0.28 \pm 0.07 \mathrm{eV}$ obtained from Ps trapping studies of the annealing of irradiated ice (Eldrup, 1976; paper in preparation by M. Eldrup, R. J. Douglas, and L. Lupton). The higher vacancy concentration will probably imply a profound change in the normally accepted theories of the defects on the hydrogen bonds in ice, which presumably explain, e.g. the dielectric properties of ice. Because of the Ps trapping results there is probably more knowledge of the vacancies and vacancy clusters in ice than in any other molecular solid.

\section{Acknowledgements}

The authors are indebted to G. Kvajić and J. H. Bilgram for providing the ice monocrystals. Thanks are also due to N. J. Pedersen for valuable technical assistance.

\section{REFERENCES}

Buxton, G. V., and others. 1975. Pulse radiolysis of aqueous solids at $76 \mathrm{~K}$. An absorption band in the infrared, [by] G. V. Buxton, H. A. Gillis and N. V. Klassen. Chemical Physics Letters, Vol. 32, No. 3, p. 533-36.

Buxton, G. V., and others. 1977. Two types of localized excess electrons in crystalline $\mathrm{D}_{2} \mathrm{O}$ ice, [by] G. V. Buxton, H. A. Gillis and N. V. Klassen. Canadian Journal of Chemistry, Vol. 55, No. 1 2, p. 2385-95.

Crawford, J. H., jr., and Slifkin, L. M., ed. 1972-75. Point defects in solids. New York, Plenum Press. 2 vols. Eldrup, M. 1976. Vacancy migration and void formation in $\gamma$-irradiated ice. Fournal of Chemical Physics, Vol. 64 ,
No. 12, p. $5283-90$.

Eldrup, M., and others. 1972. Positron lifetimes in pure and doped ice and in water, [by] M. Eldrup and O. E. Mogensen and G. Trumpy. Journal of Chemical Physics, Vol. 57, No. 1, p. 495-504.

Eldrup, M., and others. 1976 . A positron annihilation study of the annealing of electron irradiated molybdenum, [by] M. Eldrup, O. E. Mogensen and J. H. Evans. Fournal of Physics F, Vol. 6, No. 4, p. 499-521.

Eldrup, M., and others. 1978. Vacancies in HF-doped and in irradiated ice by positron annihilation techniques, by M. Eldrup and O. E. Mogensen and J. H. Bilgram. Journal of Glaciology, Vol. 21, No. 85, p. I01-13. Fletcher, N. H. I970. The chemical physics of ice. Cambridge, University Press. (Cambridge Monographs on
Physics.) Goldanskii, V. I. 1968. Physical chemistry of the positron and positronium. Atomic Energy Review, Vol. 6, No. I,
p. $3^{-1} 4^{8}$.

Henderson, B., and Hughes, A. E., ed. 1976. Defects and their structure in nonmetallic solids. New York, Plenum Press.

Hobbs, P. V. 1974. Ice physics. Oxford, Clarendon Press. 
Huber, $\mathrm{H}$., and others. 1973. Channeling of $\mathrm{H}^{+}, \mathrm{D}^{+}$, and $\mathrm{He}^{+}$in ice: surface disorder and chlorine location, [by] H. Huber, C. Jaccard and M. Roulet. (In Whalley, E., and others, ed. Physics and chemistry of ice: papers presented at the Symposium on the Physics and Chemistry of Ice, held in Ottawa, Canada, I4-1 3 August 1972. Edited by E. Whalley, S. F. Fones, L. W. Gold. Ottawa, Royal Society of Canada, p. 137-39.)

Kawa bata, K. 1976. Electron traps in irradiated crystalline ice. Fournal of Chemical Physics, Vol. 65, No. 6,

p. 2235-42.
Kirkegaard, P., and Eldrup, M. 1974. Positronfit extended: a new version of a program for analysing positron lifetime spectra. Computer Physics Comnunications, Vol. 7, No. 7, p. 40I-og.

Kirkegaard, P., and Mogensen, O. E. Unpublished. PAACFIT: a program for analysing positron annihilation angular correlation spectra. [[Risø National Laboratory, Roskilde, Denmark. Report] M-i6i5, I973.]

Milošević-Kvajić, M., and others. 1972. Angular correlation of annihilation photons in frozen aqueous solutions, [by] M. Milošević-Kvajić, O. E. Mogensen, G. Kvajić and M. Eldrup. Fournal of Chemical Physics, Vol. 56, No. 6 , p. $2567-71$.

Mogensen, O. E. Unpublished. Theory of the annihilation probability of a positronium many-electron system. [Fourth International Conference on Positron Annihilation, Helsingør, Denmark, 1976, Preprint G3,

p. G $\left.{ }_{13}-G_{1} 8.\right]$
Mogensen, O. E., and Eldrup, M. I973. Positronium interaction with defects and impurities in ice. (In Whalley, E., and others, ed. Physics and chemistry of ice: papers presented at the Symposium on the Physics and Chemistry of Ice, held in Ottawa, Canada, I $4^{-1} 8$ August 1972. Edited by E. Whalley, S. F. Jones, L. W. Gold. Ottawa, Royal Society of

Canada, p. 165-69.)
Mogensen, O. E., and Eldrup, M. 1977[a]. On the vacancy concentration in ice. Physics Letters, Vol. 6oA, No. 4,

p. $325^{-26}$.
Mogensen, O. E., and Eldrup, M. 1977[b]. Positronium Bloch function, and trapping of positronium in vacancies, in ice. Risø Report (Risø National Laboratory, Roskilde, Denmark), No. 366.

Mogensen, O. E., and others. 1971. Angular correlation of annihilation photons in ice single crystals, by O. E. Mogensen, G. Kvajić, M. Eldrup, and M. Milošević-Kvajić. Physical Review B, Third Ser., Vol. 4, No. I,

p. $7 \mathrm{I}-73$.
Petersen, K., and others. I970. Temperature dependence of the positron lifetime in $\mathrm{H}_{2} \mathrm{O}$ and $\mathrm{D}_{2} \mathrm{O}$, [by] K. Petersen, M. Eldrup and G. Trumpy. Physics Lellers, Vol. 31 A, No. 3, p. 109-1o.

Ramseier, R. O. 1967. Self-diffusion of tritium in natural and synthetic ice monocrystals. Fournal of Applied Physics, Vol. 38, No. 6, p. 2553-56.

Seidensticker, R. G., and Longini, R. L. 1969. Impurity statistics in ice. (In Riehl, N., and others, ed. Physics of ice: proceedings of the international symposium on physics of ice, Munich, Germany, September 9-14, 1968. Edited by $\mathcal{N}$. Riehl, B. Bullemer, H. Engelhardt. New York, Plenum Press, p. $47 \mathrm{I}-82$.

Stoneham, A. M. 1975. Theory of defects in solids. Oxford, Clarendon Press.

West, R. N. 1973. Positron studies of condensed matter. Advances in Physics, Vol. 22, No. 3, p. $263-383$.

Whalley, E., and others, ed. 1973. Physics and chemistry of ice: papers presented at the Symposium on the Physics and Chemistry of Ice, held in Ottawa, Canada, I $^{-18} 8$ August 1972. Edited by E. Whalley, S. F. Fones, L. W. Gold. Ottawa, Royal Society of Canada.

\section{DISGUSSION}

J. W. GLen: This represents an important revision of our understanding of the energetics of vacancies - can we be sure that what you are seeing are vacancies and not some other point defect?

M. EldRup : In our papers we list a number of reasons which we find give strong evidence that we really see vacancies. In particular there is independent evidence from radiation chemistry and self-diffusion results that vacancies in p.p.m. concentrations are present in irradiated and in pure crystals at low and high temperatures respectively. In our own annealing experiments on HF-doped and on irradiated crystals we find very long-lived components. They can only be explained as ortho-positronium trapped in rather large cavities (probably equivalent to I0-50 missing water molecules). There seems no other way these cavities can form than by clustering of vacancies. Hence, there is little doubt that vacancies are present. Another question is whether the $1.2 \mathrm{~ns}$ lifetime component can be associated with certainty with orthopositronium trapped at monovacancies. However, we find it very unlikely that positronium should instead get trapped at some unknown hole-type defect of about the same size as a vacancy (as judged from our data), and not in the vacancies which we know are present. The only other possible defects might be dislocations, but the dislocation density is probably too small for them to be detected, and it is not certain that dislocations in high concentration will trap positronium.

L. Couture: What is the size of the positronium? 
EldRUP: The same as that of the hydrogen atom, i.e. a diameter of about I $\AA$ in vacuum in the movement of the electron and positron around the centre of mass. On the other hand the centre of mass may be delocalized as it is in defect-free ice.

G. Noll: What is known about self-trapping of positronium in ice. Do you have some evidence for it in your results?

EldRUP: At low temperatures $\left(T<-\mathrm{IOO}^{\circ} \mathrm{C}\right)$ angular correlation curves show that positronium is delocalized and hence not trapped. At higher temperatures we find trapping of positronium. As mentioned, we have good reasons to believe that the trapping takes place at vacancies. However, we cannot rule out that some self-trapping takes place, although the large increase in the ortho-positronium lifetime $\tau_{3}(c .0 .7$ to $\mathrm{I} .0 \mathrm{~ns})$ is larger than you would expect for self-trapping.

K. Itagaki: Can positronium annihilation detect dislocations? If so, may not the anomalously high vacancy concentration apparently observed by positronium annihilation be caused by dislocations?

EldRUP: We do not know if dislocations can trap positronium in ice. Even if they can, the dislocation density in our ice crystals is probably below the limit of detection. In our paper we discuss the possibility that dislocation creation and movement might be the source of vacancies at low temperatures in HF-doped ice, but conclude that this mechanism is unlikely. We do not think that the vacancy concentration in ice is "anomalously" high, since in metals (and apparently also in other molecular crystals) the relative vacancy concentration is of the order of $\mathrm{IO}^{-5}$ to $\mathrm{IO}^{-4}$ or higher close to the melting point.

W. B. KaмB: If the positronium can be trapped at molecular vacancies in the ice structure, should it not also be trapped by the large cavities that are already present in the complete ice structure without any vacancies? If not, why not?

EldRup: In non-defected ice, positronium will feel a potential which has translational symmetry (apart from perturbations by the proton disorder) and will be delocalized in this potential as evidenced by the narrow central and side peaks from para-positronium in angularcorrelation curves. This is similar to the delocalization of conduction electrons in metals. A positronium atom localized (i.e. trapped) in a cavity in the perfect ice lattice would have a higher zero-point energy than in a delocalized state (of the order of $\mathrm{I} \mathrm{eV}$ ). Thus localization is not energetically favourable. However, a vacancy or a cluster of vacancies seem to provide sufficiently deep potential wells that the decrease in positronium potential energy more than compensates the increase in positronium zero-point energy when positronium is trapped in one of these defects.

J. F. Nagle: To follow up Professor Kamb's question a little further, is it possible that the randomness of the protons in ice introduces a random potential for the positronium which then gets bound into localized states in analogy to the conventional wisdom for electrons in amorphous semiconductors? And could this be an alternative explanation to trapping in vacancies?

Eldrup: At low temperatures $\left(<c .-\mathrm{IOO}^{\circ} \mathrm{C}\right)$ the angular correlation curves show that positronium is delocalized (Figs 3 and 4 ). Thus, at low temperatures the randomness of protons does not lead to localized positronium states. At higher temperatures in pure ice, and also at low temperatures in HF-doped ice, angular correlation curves reveal a component of width fwhm $=3-4$ mrad equivalent to localization of positronium within $3-4 \AA$. It seems unlikely that such localization could result from an "enhancement" of the random potential from the protons brought about by thermal vibrations or HF doping. Maybe the random proton potential can explain the difference in the relative intensity changes of the central and side peaks with increasing temperature in pure ice (Mogensen and Eldrup, 1977[b]). 